\title{
Caracterización dinámica mediante barra Hopkinson de materiales cerámicos monolíticos y compuestos
}

\author{
A. KHALIFA ${ }^{1,2}$, E. BARBERO ${ }^{1}$, P. MIRANZO ${ }^{2}$, C. NAVARRO ${ }^{1}$ \\ ${ }^{1}$ Area de Mecánica de Medios Continuos y Teoría de Estructuras. Universidad de Carlos III de Madrid. Leganés.(Madrid). \\ ${ }^{2}$ Instituto de Cerámica y Vidrio.Centro Superior de Investigaciones Científicas Arganda del Rey, (Madrid).
}

\begin{abstract}
El objetivo de este artículo es la caracterización en compresión dinámica, a alta velocidad de deformación, de materiales cerámicos de alumina, tanto monolíticos como compuestos (alúmina/SiC-plaquetas, 8 y 30\% en volumen), utilizando el dispositivo denominado barra Hopkinson. Para resolver los problemas originados por la elevada dureza de estos materiales se han ensayado diferentes soluciones, encontrándose como óptima la intercalación entre barra y probeta de discos metálicos desechables del mismo material de la barra. Los datos obtenidos de las medidas permitieron obtener la resistencia mecánica de los materiales ensayados en condiciones dinámicas. La incorporación del 30\% en volumen de SiC aumenta la deformación obtenida de la matriz.
\end{abstract}

Palabras claves: materiales compuestos de matriz cerámica, alúmina, carburo de silicio, impacto, compresión dinámica, barra Hopkinson.

\section{Dynamic characterization of monolithic and composite ceramic materials using Hopkinson bar}

The mechanical behaviour of monolithic and composite ceramic materials was analysed under impact conditions, using the Hopkinson bar to study the response and failure modes. The materials considered were alumina $\left(\mathrm{Al}_{2} \mathrm{O}_{3}\right)$ and silicon carbide platelet / alumina matrix $\left(\mathrm{Al}_{2} \mathrm{O}_{3} / \mathrm{SiC}_{\mathrm{pl}}\right)$ composites. Because of the high hardness of ceramics, modifications of the conventional Hopkinson bar device were done to prevent the damage of the bars'surface. Stress-strain curves obtained from the tests showed a significant increase in strain values when $30 \%$ vol. of $\mathrm{SiC}$ platelet are included in the matrix.

Key words : Ceramic matrix composites, Alumina, Silicon carbide, Impact, dynamic compression, Hopkinson bar

\section{INTRODUCCIÓN}

Los materiales cerámicos son buenos candidatos para aplicaciones estructurales debido a su buen comportamiento frente al desgaste y a la corrosión, a su baja densidad, a su bajo coeficiente de dilatación térmica y a sus buenas propiedades térmo-mecánicas (1-2). A la vista de las características anteriores, se vislumbran las posibles aplicaciones de los materiales cerámicos avanzados: herramientas de corte de alta velocidad para operaciones de acabado o de mecanizado, prótesis, blindajes, componentes de motores y en sistemas de protección térmica para vehículos espaciales. Dentro del campo del blindaje, se requieren materiales cerámicos de elevada dureza y alta resistencia mecánica (3-4). El material más empleado en estas aplicaciones es la alúmina monolítica, aunque para mejorar su resistencia mecánica se puede añadir como refuerzo una segunda fase, tal como el carburo de silicio (2,5-8).

Los trabajos relativos al estudio del comportamiento mecánico de materiales cerámicos se han centrado, en general, en ensayos estáticos. De hecho, existe escasa información sobre el comportamiento dinámico de los materiales cerámicos (8-14), en particular, para velocidades de deformación muy elevadas. La información se reduce a ensayos a velocidades de deformación medias. Este trabajo se ha dirigido al estudio del comportamiento de los materiales cerámicos frente a cargas dinámicas a altas velocidades de deformación, en concreto en compresión. Para ello se ha seleccionado un dispositivo muy empleado en el estudio de materiales metálicos, denominado barra Hopkinson, $(14,15)$. Se han estudiado materiales de alúmina monolítica y de alúmina reforzada con $8 \%$ y $30 \%$, en volumen, de plaquetas de carburo de silicio.

\section{FABRICACIÓN Y MÉTODO DE CARACTERIZACIÓN MICROESTRUCTURAL}

Los materiales empleados en el estudio mecánico se han obtenido por medio de un método de procesado clásico de polvos cerámicos. Las características de los polvos de partida empleados para la fabricación de los materiales se recogen en la tabla I.

TABla I. CARACTERÍsticas de los POlvos DE PARTIDA

\begin{tabular}{|l|c|c|}
\hline & $\mathrm{Al}_{2} \mathrm{O}_{3}$ & $\mathrm{SiC}_{\mathrm{pl}}$ \\
\hline Tamaño $(\mu \mathrm{m})$ & 0,4 & 16 \\
\hline Superficie especifica $\left(\mathrm{m}^{2} / \mathrm{g}\right)$ & 7,5 & 0,7 \\
\hline Fabricante & $\begin{array}{c}\text { Martinswerk } \\
\text { Alemania }\end{array}$ & $\begin{array}{c}\text { C-Axis Tech. } \\
\text { Canadá }\end{array}$ \\
\hline
\end{tabular}


Las mezclas de polvos de $\mathrm{Al}_{2} \mathrm{O}_{3}$ y plaquetas de $\mathrm{SiC}(8 \%$ y $30 \%$ en volumen) se homogeneizaron en un molino de atricción utilizando alcohol isopropílico como medio líquido dispersante y bolas de $\mathrm{Al}_{2} \mathrm{O}_{3}$ como medio de molienda. La suspensión homogénea de los distintos componentes se secó por agitación mecánica para evitar que las partículas se segregaran y precipitaran. Los polvos obtenidos se tamizaron a un tamaño de $100 \mu \mathrm{m}$ y se prensaron en caliente (FCT Alemania) en atmósfera de argón, aplicando una presión uniaxial de $50 \mathrm{MPa}$. Este proceso se realizó a $1500^{\circ} \mathrm{C}$ durante 30 min para la material de $\mathrm{Al}_{2} \mathrm{O}_{3}$ monolítica y el material con $8 \%$ en volumen de plaquetas, y a $1550^{\circ} \mathrm{C}$ durante $60 \mathrm{~min}$ para el material con $30 \%$ en volumen de plaquetas de SiC. Se obtuvieron materiales con densidades siempre superiores al $99 \%$ de la densidad teórica, calculada utilizando el principio de Arquímedes.

Para todos los materiales se obtuvieron discos de $50 \mathrm{~mm}$ de diámetro y $7 \mathrm{~mm}$ de espesor. Estos discos fueron rectificados y mecanizados con una broca cilíndrica hueca recubierta de diamante para obtener probetas cilíndricas de $8.8 \mathrm{~mm}$ de diámetro $\times 5 \mathrm{~mm}$ de espesor. Se fabricaron, para su ensayo mecánico, 5 probetas de cada material, de forma que su eje era paralelo a la dirección de prensado.

La microestructura de los materiales se analizó mediante microscopía óptica de luz reflejada (MOLR). Para el estudio de la microestructura los materiales se pulieron secuencialmente con polvo de diamante de 6,3 y $1 \mu \mathrm{m}$, hasta conseguir un acabado especular en las muestras. En la Figura 1 se muestra la micrografía de la alumina reforzada con un $30 \%$ en volumen de plaquetas de $\mathrm{SiC}$, en la que se observa que existe una buena dispersión del refuerzo en la matriz.

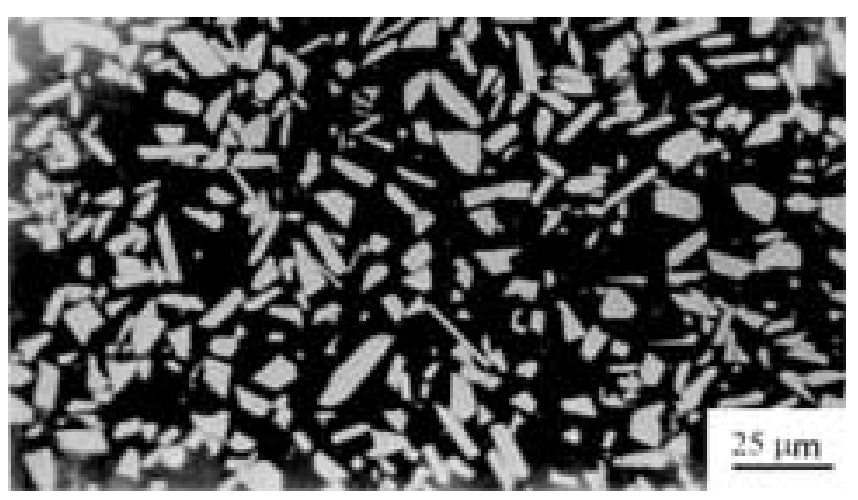

Figura 1. Fotografía obtenida mediante MOLR del material de $\mathrm{Al}_{2} \mathrm{O}_{3} / 30 \% \mathrm{SiC}_{\mathrm{p}}$. Sección $\perp$ al eje de prensado.

Para el análisis de las superficies de fractura de las probetas ensayadas en la barra Hopkinson, se utilizó microscopía electrónica de barrido (MEB).

\section{CARACTERIZACION MECÁNICA}

El comportamiento frente a cargas dinámicas de los materiales fabricados se analizó en compresión dinámica a alta velocidades de deformación. Este ensayo no está normalizado en el caso de los materiales cerámicos, lo que ha obligado al desarrollo de una metodología propia para la realización del mismo. El equipo que se empleó es el denominado barra Hopkinson de compresión.

El dispositivo de ensayo contempla, tal como se muestra en la Figura 2, la utilización de dos barras de acero inoxidable (incidente y transmisora), con sus caras perfectamente planas y ortogonales a sus ejes, entre las que se coloca una probeta cilíndrica del material a ensayar.



Figura 2. Esquema del dispositivo de compresión de barra Hopkinson.

Sobre la barra incidente se hace impactar un proyectil cilíndrico impulsado por aire comprimido. El impacto origina una onda de compresión que se propaga en la barra hasta llegar a la probeta, donde parte se transmite a la segunda barra y parte se refleja. Dichas barras están instrumentadas mediante bandas extensométricas, las cuales constituyen la base física de las medidas $(14,15)$. De la señal de dichas bandas se obtiene un registro de las ondas incidente, transmitida y reflejada (Figura 3). En el equipo disponible las barras son de acero de $1 \mathrm{~m}$ de longitud y $22 \mathrm{~mm}$ de diámetro. Los ensayos se realizaron a temperatura ambiente, a una velocidad de deformación de aproximadamente $500 \mathrm{~s}^{-1}$.



Figura 3. Ondas incidente, reflejada y transmitida. Ensayo de compresión sobre $\mathrm{Al}_{2} \mathrm{O}_{3} / 30 \% \mathrm{SiC}_{\mathrm{pl}}$.

Tal como se observa en la Figura 3, la onda reflejada presenta unas características diferentes a las que se obtienen en un ensayo de compresión sobre materiales metálicos(15), presentando un acusado descenso. La razón de este comportamiento está asociada, sin duda, a la elevada fragilidad y rigidez de los materiales cerámicos, que hace que el material se fracture catastróficamente. 


\section{RESULTADOS Y DISCUSIÓN}

La barra Hopkinson fue originariamente desarrollada para el ensayo de materiales metálicos. En la realización de ensayos de compresión sobre materiales cerámicos aparecen problemas debido a la elevada resistencia mecánica, rigidez y dureza de estos materiales. Sobre las barras se producen daños debido a un fenómeno de plastificación localizado, lo que obliga a rectificar las superficies de las barras en contacto con la probeta después de cada ensayo. Además, si se emplea la técnica convencional para la obtención de la deformación que sufre el material analizado, se obtiene un valor sobrestimado dado que parte corresponde a la deformación en la barra Hopkinson y no a la de la probeta.

Para evitar estos dos problemas se ha propuesto soluciones basadas en discos cerámicos o metálicos que protegen las barras (10). Se han probado materiales con diferentes propiedades mecánicas (en concreto dureza) para la fabricación de los discos, tales como alúmina, carburo de silicio, intermetálico titanio-aluminio y acero de alta dureza, realizando diferentes ensayos sobre alúmina monolítica. En los tres primeros casos se observó que se producía la rotura del disco debido a su fragilidad. Además, esta solución plantea el problema de la reflexión de la onda incidente en la superficie de contacto disco protector/barra debido a las diferencias de impedancia entre discos y barra, por lo que los resultados obtenidos de estos ensayos no se consideraron válidos. El mejor comportamiento lo mostró el acero de alta dureza, aunque se producía una pequeña indentación sobre el disco. En este caso, el problema de reflexión en la superficie de contacto no existe ya que se utiliza el mismo material de las barras, y no se produce la rotura de los discos. Este efecto se analizó realizando un ensayo en vacío (sin probeta) con y sin discos. Como se observa en la Figura 4, sólo existe onda incidente y transmitida, por lo que la presencia de estos discos no afecta a la propagación de las ondas. Dado que no se ha establecido ningún mecanismo para evitar la indentación de la probeta sobre el disco, la medida de la deformación obtenida con este sistema está sobrestimada.

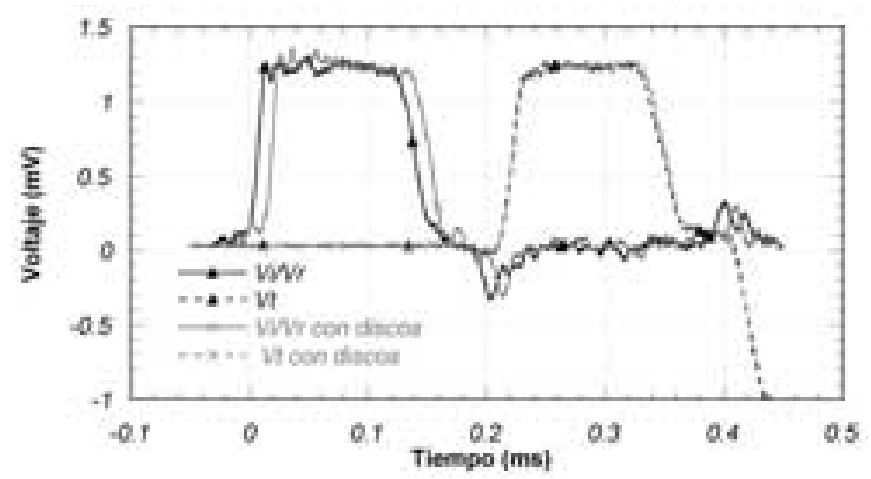

Figura 4. Ondas incidente, transmitida y reflejada en un ensayo en vacío con y sin discos de protección.

A la vista de los resultados anteriores se propuso el empleo de discos metálicos del mismo material y diámetro que las barras según se muestra en el esquema en la Figura 5. Aunque los discos quedan dañados, pueden ser reparados con mayor facilidad que la totalidad de la barra.

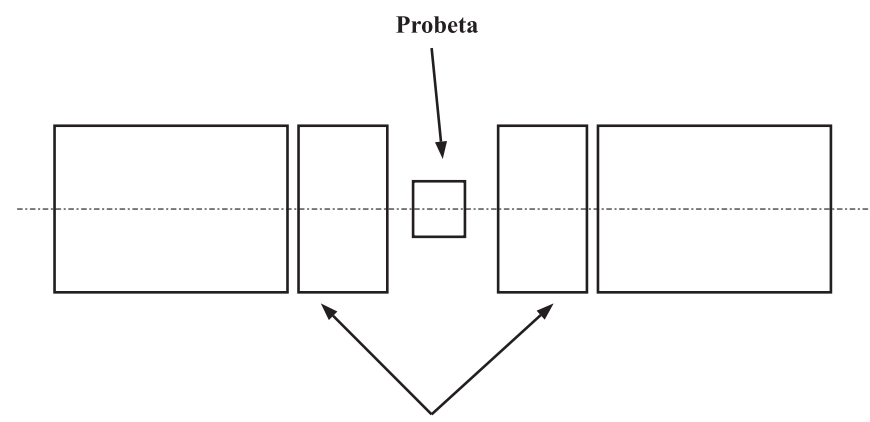

Discos de protección

Figura 5. Esquema de situación de los discos de protección

A partir de los registros de las bandas extensométricas (Figura 3), y empleando las técnicas estándar $(15,16)$, se obtuvo la variación de la tensión y de la deformación con el tiempo, como se muestra en las Figuras 6 y 7 para uno de los materiales analizados.

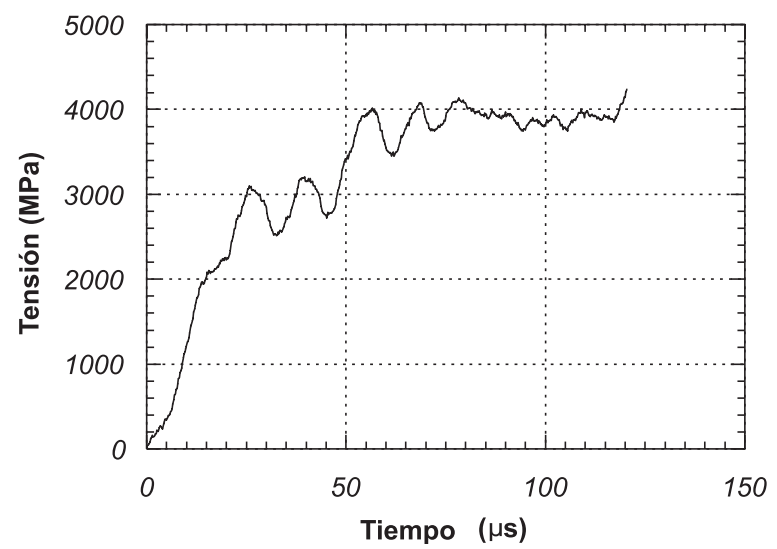

Figura 6. Variación de la tensión con el tiempo en una probeta de $\mathrm{Al}_{2} \mathrm{O}_{3} / 30 \% \mathrm{SiC}_{\mathrm{pl}}$



Figura 7. Variación de la deformación con el tiempo en una probeta de $\mathrm{Al}_{2} \mathrm{O}_{3} / 30 \% \mathrm{SiC}_{\mathrm{pl}}$ 
Los valores de deformación medidos durante el ensayo son muy altos para los materiales cerámicos, esto se explica porque la técnica para calcular la deformación de un material en la barra Hopkinson se basa en una medida indirecta de la distancia entre las barras incidente y transmisora $(14,15)$. En los materiales cerámicos esta medida se ve afectada por el daño que las probetas producen sobre las barras. De esta forma, las curvas deformación-tiempo (Figura 7) deben considerarse como una primera aproximación, válida para establecer comparaciones entre materiales. Para la medición directa de la deformación del material ensayado se propone el empleo de galgas extensométricas directamente adheridas a la probeta.

A partir de las curvas tensión-tiempo y deformación-tiempo, fue posible construir las curvas tensión-deformación verdadera, para los tres diferentes materiales estudiados, $\mathrm{Al}_{2} \mathrm{O}_{3^{\prime}}$ $\mathrm{Al}_{2} \mathrm{O}_{3} / 8 \% \mathrm{SiC}_{\mathrm{pl}}, \mathrm{y} \mathrm{Al}_{2} \mathrm{O}_{3} / 30 \% \mathrm{SiC}_{\mathrm{pl}}$. Una curva representativa de cada material se muestra en la Figura 8. A partir de dichas curvas es posible estimar la resistencia mecánica a compresión dinámica de los tres materiales, obteniéndose 4000, 3800, $3000 \mathrm{MPa}$ para $\mathrm{Al}_{2} \mathrm{O}_{3^{\prime}}, \mathrm{Al}_{2} \mathrm{O}_{3} / 8 \% \mathrm{SiC}_{\mathrm{pl}}$ y $\mathrm{Al}_{2} \mathrm{O}_{3} / 30 \% \mathrm{SiC}_{\mathrm{pl}^{\prime}}$ respectivamente. La incorporación de un $8 \%$ en vol de plaquetas de $\mathrm{SiC}$ no afecta el valor de la resistencia mecánica a altas velocidades de deformación de la alúmina. Un aumento del contenido de plaquetas hasta un $30 \%$ en volumen produce una disminución drástica de la resistencia mecánica del material. En este tipo de materiales, se ha observado un descenso similar de la resistencia mecánica en flexión en condiciones estáticas al añadir plaquetas de $\mathrm{SiC}$ (5). Este descenso se interpreta porque, las diferencias en los coeficientes de expansión térmica de la alumina y de carburo de silicio producen un campo de tensiones residuales, de forma que el defecto crítico en estos materiales aumenta.

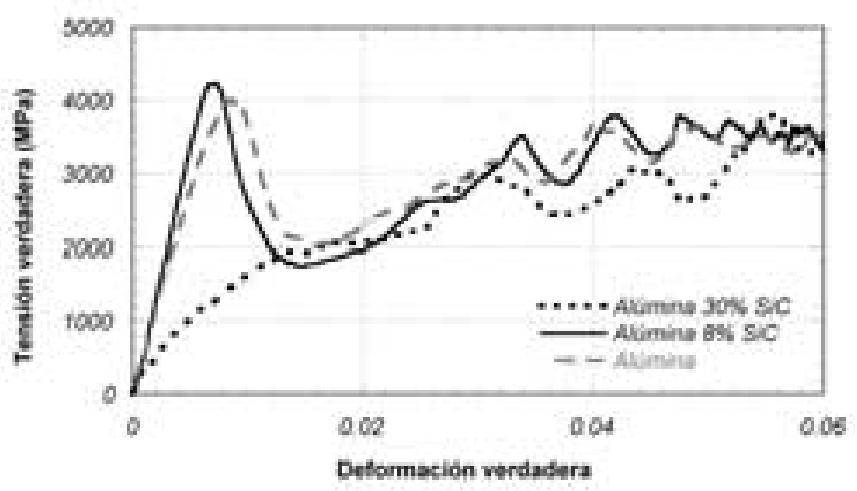

Figura 8. Curvas tensión-deformación obtenidas para los materiales de $\mathrm{Al}_{2} \mathrm{O}_{3}, \mathrm{Al}_{2} \mathrm{O}_{3} / 8 \% \mathrm{SiC}_{\mathrm{pl}}$ y $\mathrm{Al}_{2} \mathrm{O}_{3} / 30 \% \mathrm{SiC}_{\mathrm{pl}}$.

Tal como aparece en la figura 8, el comportamiento mecánico de los materiales de $\mathrm{Al}_{2} \mathrm{O}_{3}$ y $\mathrm{Al}_{2} \mathrm{O}_{3} / 8 \% \mathrm{SiC}_{\mathrm{pl}}$ es muy semejante entre sí. Sin embargo, la curva tensión-deformación del material reforzado con un $30 \%$ en volumen de plaquetas es muy diferente de los otros dos materiales, encontrándose que posee una menor rigidez. El comportamiento diferente de este material, se puede explicar estudiando las superficies de fractura de los materiales, mostradas en las Figuras 9-11.

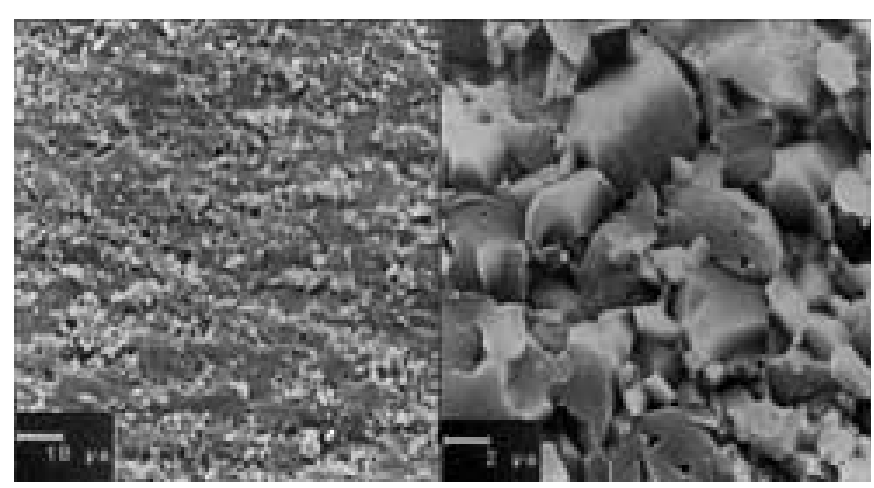

Figura 9. Superficie de fractura en $\mathrm{Al}_{2} \mathrm{O}_{3}$.

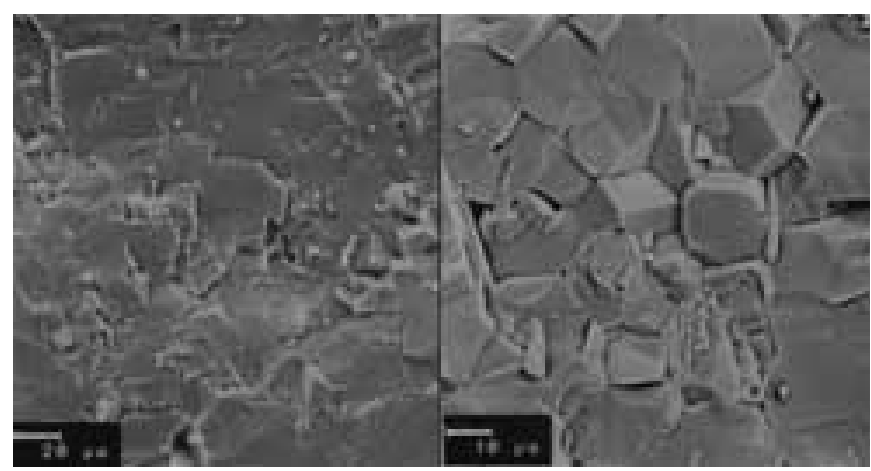

Figura 10. Superficie de fractura en $\mathrm{Al}_{2} \mathrm{O}_{3} / 8 \% \mathrm{SiC}_{\mathrm{pl}}$.

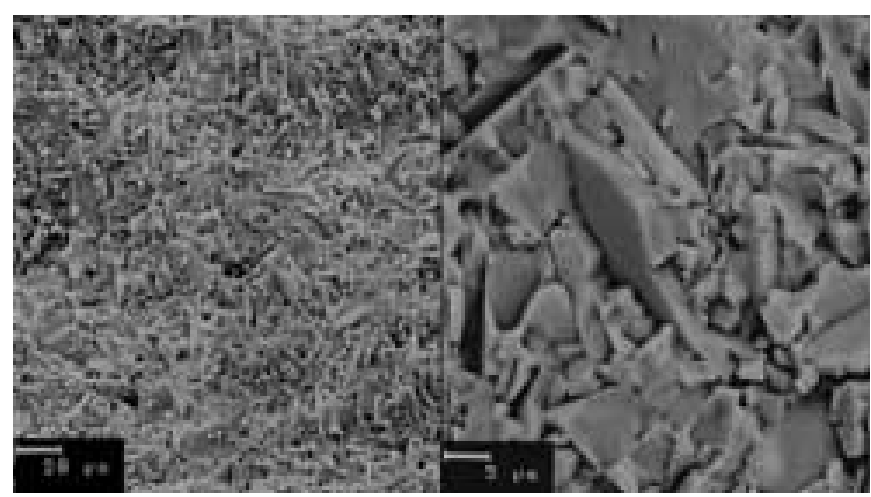

Figura 11. Superficie de fractura en $\mathrm{Al}_{2} \mathrm{O}_{3} / 30 \% \mathrm{SiC}_{\mathrm{pl}}$.

El material monolítico de $\mathrm{Al}_{2} \mathrm{O}_{3}$ muestra un modo de fractura mixto transgranular / intergranular. $\mathrm{Al}$ añadir un $8 \%$ vol. de plaquetas de $\mathrm{SiC}$, la fractura es totalmente transgranular, mientras que cuando el contenido de plaquetas es del $30 \%$ en volumen el modo de fractura cambia a totalmente intergranular, observándose arranque de algunas plaquetas, Figura 11. Este tipo de fractura, generalmente asociada con el mecanismo de reforzamiento por puenteo de la grieta, también ha sido observado en estos materiales en ensayos de flexión en condiciones estáticas (5). Esto mecanismo de reforzamiento produce un aumento de la energía de fractura y conduce a un tipo de rotura no-catastrófico en el que la deformación de rotura aumenta (2). 


\section{CONCLUSIONES}

Se ha utilizado el dispositivo de barra Hopkinson de compresión para estudiar el comportamiento mecánico frente a cargas dinámicas de materiales monolíticos de $\mathrm{Al}_{2} \mathrm{O}_{3}$ y materiales compuestos de $\mathrm{Al}_{2} \mathrm{O}_{3} / \mathrm{SiC}_{\mathrm{p}}$

Debido a la elevada dureza de los materiales cerámicos ensayados, las técnicas clásicas que se emplean en los materiales metálicos no son válidas. Por ello, el valor de deformación medido está sobrestimado.

Aunque no es posible hablar de valores absolutos, este método nos ha permitido observar diferencias importantes en el comportamiento mecánico de estos materiales Un aumento del porcentaje de plaquetas de $\mathrm{SiC}$ hasta $30 \%$ en volumen produce una reducción en la rigidez del material.

\section{BIBLIOGRAFÍA}

1. W.D. Kingery, H.K. Bowen y D.R. Uhlman, Introduction to Ceramics, $2^{\underline{a}}$ edición. Jhon Wiely and Sons (New York, EE.UU.), (1976)

2. K.K. Chawla, "Ceramic Matrix Composites", $1^{\text {st }}$ ed. Chapaman \& Hall, (1993).

3. J.D. Viechnicki, M.J. Slavin y M.I. Kliman, "Developement and Current Status of Amor Ceramics", Ceramic Bulletin, vol. 70, No. 6, 1035-1039 (1991).

4. W.J. Cantwell y J. Morton, "The impact resistance of composite materials- a review", composites, 9, p 347-362 (1991).

5. M. Belmonte, J.S. Moya, P. Miranzo, D. Nguyen, J. Dubois, y G. Fantozzi, "Fracture Behavior of $\mathrm{Al}_{2} \mathrm{O}_{3}$ /SiC-Platelet Composites", J. Mater. Res., vol. 11, 2528-2535 (1996).
6. P. Miranzo y J.S. Moya, "Reforzamiento de Materiales Cerámicos y Vitreos por Fibras", Bol. Soc. Esp. Ceram. Vidr. 27, 3, 145-151 (1988).

7. N. Claussen, Ceramic "Platelet Composites". pp 1-12 en Structural Ceramics Processing. Microstructure and Properties. Proceedings del $11^{\text {th }}$ RISInternational Symposium (Roskilde, Dinamarca, Septiembre 1990). Editado por J.J Bentzen, J.B. Bilde-Srensen, N. Christiansen, A. Horsewell y B. Ralph, RIS National Laboratory (Dinamarca), 1990.

8. Y. Takagi y A.S. Kobayashi, "Dynamic Fracture Responses of Alumina, Silicon Nitride and Silicon Carbide Whisker / Alumina Matrix", Fracture Mechanics of Ceramics, vol. 9, 133-145 (1992).

9. M. Adams y G. Sines, "Methods for Determining the Strength of Brittle Materials in Compressive Stress States", Journal of Testing and Evaluation, 4, [6], 383-396 (1976).

10. J. Lankford, E. Anderson, A. Nagy, J. Walker, A. Nicholls y R. Page, "Inelastic Response of Confined Aluminium Oxide under Dynamic Loading Conditions", Journal of Materials Science, 33, 1619-1625 (1998).

11. E. Barbero, J. Fernández-Sáez y C. Navarro, "Dynamic Behaviour of Ceramic Matrix Composites Processed by Dry Pressing", 12 th Dymat Technical Meeting Coimbre-Portugal, October 1999, pp.65-70.

12. K. H. Yang, y A. S. Kobayashi, "Dynamic Fracture Responses of Alumina and Two Ceramic Composites", J. Am. Ceram. Soc. 73[8] 2309-15 (1990).

13. E. Anderson, Jr. O'donoghue, J. Lankford y J. Walker, "Numerical Simulations of SHPB Experiments for the Dynamic Compressive Strength And Failure Of Ceramics", Inter. J. Fracture 55: 193-208 (1992).

14. J. Rodríguez, "Análisis y Desarrollo de Metologías para la Obtención de Propiedades Mecánicas de Materiales a Altas Velocidades de Deformación y a Alta Temperatura", Tesis Doctoral, E.T.S.I. Caminos de la Universidad de Complutense de Madrid (1993).

15. Mํ..H. Miguélez, "Caracterización Dinámica de Materiales Avanzados a Alta Velocidad de Deformación y a Elevada Temperatura", Tesis Doctoral, Universidad Carlos III de Madrid (1998).

Recibido: 06.08 .01

Aceptado: 15.11 .01 